\title{
Constitutional Supercooling in Czochralski Growth of Heavily Doped Silicon Crystals
}

\author{
J. Friedrich ${ }^{a, b, *}$, L. STOCKMEIER $^{a}$ AND G. MÜLleR ${ }^{b}$ \\ ${ }^{a}$ Fraunhofer THM, Am St.-Niclas-Schacht 13, 09599 Freiberg, Germany \\ ${ }^{b}$ Fraunhofer Institut IISB, Schottkystr. 10, 91058 Erlangen, Germany
}

\begin{abstract}
This study analyses the phenomenon of constitutional supercooling, which is one of the major problems in industrial growth of heavily doped $\left(>10^{20}\right.$ atoms $\left./ \mathrm{cm}^{3}\right)$ silicon crystals by the Czochralski technique. The systematic study is based on theoretical models and experimental data considering the effect of three important dopants (B, P, and As) in dependence of the relevant growth parameters for the Czochralski process. Based on these results, conclusions will be drawn for the stability limits of the Czochralski growth of dislocation-free heavily doped silicon crystals in dependence of the doping species and their concentration.
\end{abstract}

DOI: $10.12693 /$ APhysPolA.124.219

PACS: 81.10.Aj, 61.72.S-, 61.72.uf, 81.10.Fq

\section{Introduction}

An important application of silicon crystals is their use for power electronic devices. Certain vertically structured power electronic devices need silicon wafers with an electrical resistivity in the lower limit of $1-5 \mathrm{~m} \Omega \mathrm{cm}$. This relatively low resistivity is required in order to minimize the switching losses and thus to increase the efficiency of the electronic devices [1]. According to Fig. 1 an elec-

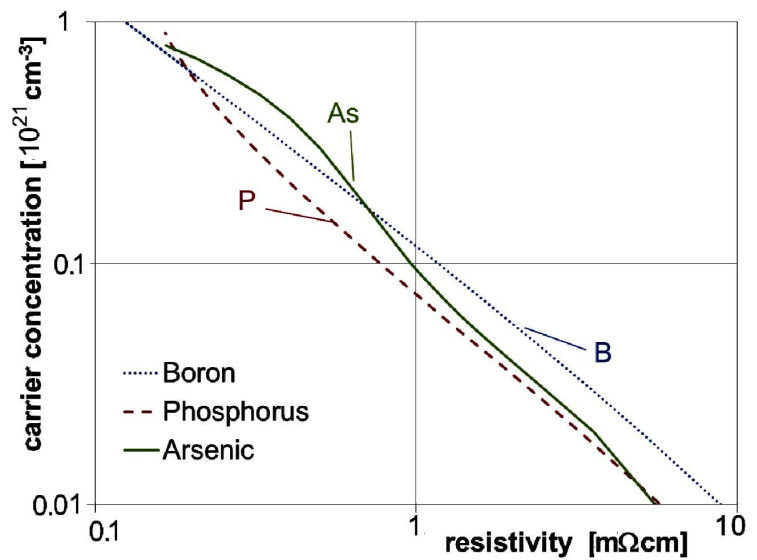

Fig. 1. Carrier concentration versus electrical resistivity in silicon doped with boron, phosphorus and arsenic. The curves were calculated according to the semi standard SEMI MF723-0307 [2].

trical resistivity of less than $5 \mathrm{~m} \Omega \mathrm{cm}$ corresponds to a carrier concentration exceeding $1 \times 10^{19} \mathrm{~cm}^{-3}$, i.e. a doping concentration higher than $1 \times 10^{19}$ atoms $/ \mathrm{cm}^{3}$. Such heavily doped silicon crystals can only be grown by the

\footnotetext{
*corresponding author; e-mail:

jochen.friedrich@iisb. fraunhofer.de
}

Czochralski (CZ) technique because these high doping levels cannot be achieved by the floating zone method. Heavy doping of silicon in $\mathrm{CZ}$ is commonly realized by adding boron, phosphorus, or arsenic to the silicon melt by special sophisticated techniques, e.g. [3-5] which are differing considerably from the doping techniques used for standard or shallow doping levels in $\mathrm{CZ}$ growth of silicon. The typical resistivity ranges which are feasible today are $0.5-20 \mathrm{~m} \Omega \mathrm{cm}$ for boron, $1-2 \mathrm{~m} \Omega \mathrm{cm}$ for phosphorus, and 2-10 $\mathrm{m} \Omega \mathrm{cm}$ for arsenic [6].

It is well known in the literature that the probability of the occurrence of dislocations and grain boundaries (called "structure loss" by CZ growers) is strongly increased in $\mathrm{CZ}$ growth by high doping concentrations [6-15]. One major origin for the increased occurrence of structure loss is considered to be a morphological instability of the solid-liquid interface [16]. This phenomenon which is illustrated by the example in Fig. 2 can be caused by the so-called constitutional supercooling. It originates from the strong enrichment of the dopant concentration in front of the solid-liquid interface (due to segregation) and the resulting reduction of the liquidus temperature by several degrees (shown quantitatively below).

The goal of this paper is to present a systematic study of the phenomenon of constitutional supercooling, i.e. morphological instability of the interface in $\mathrm{CZ}$ growth of heavily doped silicon. The study will be based on theoretical models and experimental data considering various material properties of the different doping elements and pulling parameters. Based on the results, conclusions will be drawn for stability limits for the $\mathrm{CZ}$ growth of dislocation-free heavily doped silicon crystals in dependence of the doping species and their concentration.

\section{Constitutional supercooling}

\subsection{Phenomenological description}

The segregation coefficient $k$ is defined by the ratio of the concentration of the solute in the crystal $C_{S}$ to its concentration in the melt $C_{L}$ (see Fig. 3): 


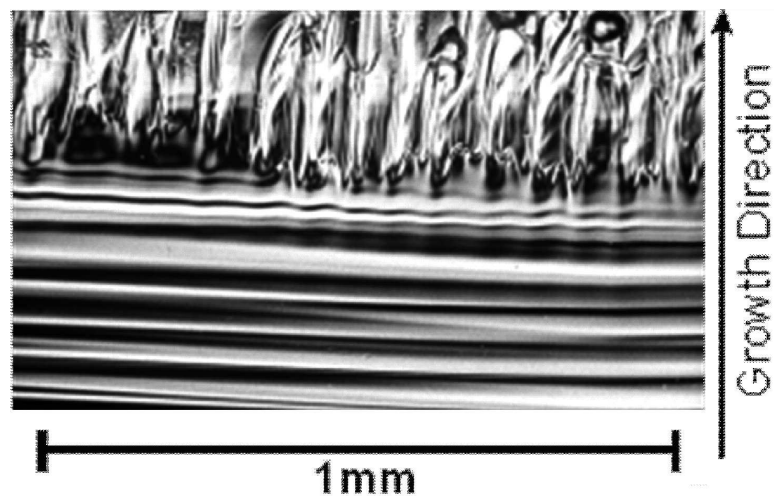

Fig. 2. Etched longitudinal section of a silicon crystal heavily doped with As $\left(1 \times 10^{19}\right.$ at. $\left./ \mathrm{cm}^{-3}\right)$ grown in a laboratory setup by the floating zone method [17].

$$
k=\frac{C_{S}}{C_{L}} .
$$

For all relevant dopants of $\mathrm{Si}$ (e.g. B, P, and As) $k$ is smaller than unity $(k<1)$. This means that the dopant concentration $C_{S}$ which is incorporated into the crystal is smaller compared to the concentration $C_{L}$ in the melt. In this case the solute is rejected from the growing interface and an enrichment of the solute in the melt will take place during crystal growth as shown by the pile up of the solute concentration $C_{L}(x)$ in Fig. 4a. A so-called solute boundary layer is formed with a thickness $\delta$ according to the phase diagram (Fig. 3). This locally depending solute concentration $C_{L}(x)$ results in a locally depending liquidus temperature $T_{e}^{\prime}(x)$ which is plotted in Fig. 4b versus the distance $x$ from the interface in the same scaling of $x$ as the plot $C_{L}(x)$ above (Fig. 4a). Additionally two possible temperature profiles $T^{\prime}(x)$ and $T^{\prime \prime}(x)$ are shown in Fig. 4b which may be given by two different designs of the hot zone or the heating conditions in a certain $\mathrm{CZ}$ growth setup.

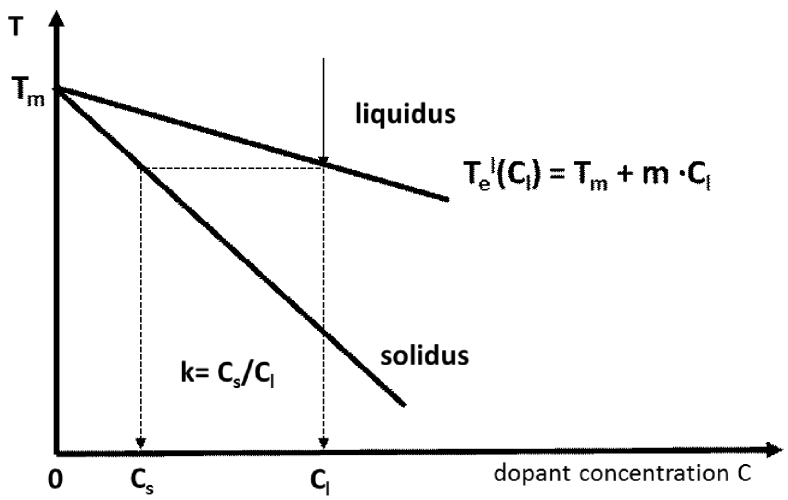

Fig. 3. Liquidus and solidus temperatures versus concentration of a dopant in the solid $\left(C_{S}\right)$ and in the liquid $\left(C_{L}\right) . \quad T_{\mathrm{m}}=$ melting point of pure silicon $\left(C_{S}=\right.$ $\left.C_{L}=0\right) . m=$ slope of liquidus line $T_{e}^{\prime}\left(C_{L}\right) . k=C_{S} / C_{L}$ is the definition of the segregation coefficient.

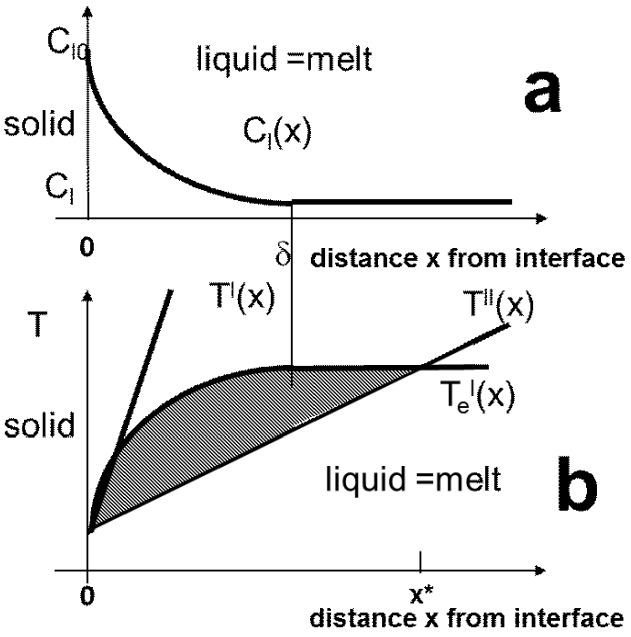

Fig. 4. (a) Concentration $C_{L}(x)$ of a dopant in the melt versus distance $x$ from the growth interface. $C_{\mathrm{L} 0}=$ concentration in the melt at the interface $(x=0) . \delta$ is the width of the boundary layer. (b) Liquidus temperature $T_{e}^{\prime}(x)$ corresponding to the concentration profile $C_{L}(x)$ plotted in (a) versus distance $x$ from the growth interface, plotted in the same scaling as in (a). The temperature profiles $T^{\prime}(x)$ and $T^{\prime \prime}(x)$ are two cases which are discussed in the text. The shaded area marks the region where the melt is "constitutionally supercooled" in front of the growth interface reaching up to position $x^{*}$ in the case of temperature profile $T^{\prime \prime}(x)$.

Constitutional supercooling occurs in the case of temperature profile $T^{\prime \prime}(x)$ because the actual melt temperature $T^{\prime \prime}(x)$ is smaller than the liquidus temperature $T_{e}^{\prime}(x)$. The region where the melt is constitutionally supercooled is marked by the shaded area in Fig. $4 \mathrm{~b}$. It reaches from the interface until position $x^{*}$. This range extends a bit more than the extension of the boundary layer $\left(x^{*}>\delta\right)$. In this situation the interface is morphologically unstable because any fluctuation forms a protuberance of the interface which grows into the supercooled melt region. This growth again increases the solute concentration in the melt by segregation and hence lowers the liquidus temperature $T_{e}^{\prime}$. This effect amplifies again the growth of the protuberance. It can be theoretically shown that a number of such projections will be formed in a close packed array separated by distances which are determined by the local transport conditions [18]. The regions between the projections become progressively richer in solute and will finally be included in the crystal. This can be observed in the experiments by a breakdown of the regular interface (compare Fig. 2). It is typically accompanied by the formation of various crystal defects which cause for example the "structure loss" in CZ growth of silicon.

Constitutional supercooling can be avoided if hot zone and heating conditions are selected to result in a temperature profile like $T^{\prime}(x)$ in Fig. $4 \mathrm{~b}$ or any profile with $T(x)>T^{\prime}(x)$, i.e. with a larger temperature gradient $G_{L}$. 
These conditions will be discussed in the following sections in detail for the $\mathrm{CZ}$ growth of silicon heavily doped with $\mathrm{B}, \mathrm{P}$, and As.

\subsection{Mathematical models for a constitutional stability limit}

The theoretical description of constitutional supercooling goes back to the pioneering works of Tiller et al. [19] for purely diffusive species transport in the melt and of Hurle [20] for convective mixing in the melt. According to their theory the ratio of the actual temperature gradient in the melt $G_{L}$ to the growth velocity $V$ must exceed a critical value which is a function of the segregation coefficient $k$, the slope $m$ of the liquidus temperature $T_{e}^{\prime}(x)$, the solute concentration $C_{L}$ in the melt and the diffusion coefficient $D$ of the solute in the melt.

\subsubsection{Purely diffusive species transport}

in the melt-Tiller's formula

For the case of purely diffusive species transport in the melt the stability criterion is defined in Eq. (2) according to Tiller et al. [19]:

$$
\frac{G_{L}}{V} \geq \frac{(1-k)(-m) C_{L}}{k D} .
$$

The temperature gradient $G_{L}$ in the melt in front of the interface can be related to the temperature gradient $G_{S}$ in the crystal at the interface by the conservation law of the heat fluxes at the interface

$$
\lambda_{S} G_{S}=\lambda_{L} G_{L}+V L \rho .
$$

$\lambda_{S}, \lambda_{L}$ are the thermal conductivities of solid (crystal) and liquid (melt), $L$ - the latent heat (heat of crystallization), $\rho$ - the density of the melt.

The slope $m=\Delta T_{L} / \Delta C_{L}$ in Fig. 2 can be estimated according to Thurmond and Kowalchik [21] for diluted alloys (like doping concentrations) by

$$
m=\frac{T_{\mathrm{m}}^{2} R}{L}=8.26 \times 10^{-27}\left[\frac{\mathrm{Km}^{3}}{\text { atoms }}\right] .
$$

$T_{\mathrm{m}}$ is the melting point temperature of the pure material (here $\mathrm{Si}$ ), $R$ - the gas constant and $L$ - the heat of crystallization. According to Hurle [20] this estimation of the slope $m$ by Eq. (2) has proven to be accurate enough for all dopants in the case of a rough, non-facetted interface, i.e. all crystal orientations except the $\langle 111\rangle$.

By introducing Eqs. (1) and (3) into Eq. (2) a stability criterion can be defined which relates the ratio of the axial temperature gradient in the solid $G_{S}$ and the growth rate $V$ to the doping level in the melt $C_{L}$. For simplicity it is assumed that $\mathrm{V}$ is equal to the pulling speed in $\mathrm{Cz}$ crystal growth, although the actual growth rate can be slightly higher than the pulling speed.

$$
\frac{G_{S}}{V}=\frac{\lambda_{L}}{\lambda_{S}} \frac{(1-k)(-m)}{k D} C_{L}+\frac{L \rho}{\lambda_{S}} .
$$

Results for the stability limit for $\mathrm{CZ}$ growth of silicon are shown in Fig. 5. In this figure the critical temperature gradient $G_{S}$ calculated from Eq. (5) for boron, phosphorus, and arsenic in the purely diffusive case is shown as dashed lines in dependence of the doping concentration $C_{L}$ in the melt for a pulling speed $V=1 \mathrm{~mm} / \mathrm{min}$.

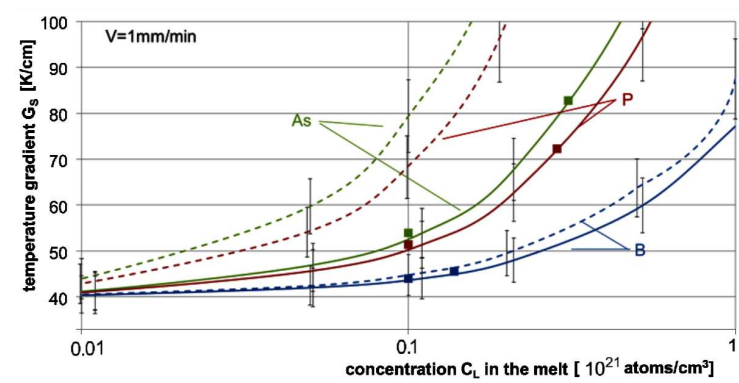

Fig. 5. Critical axial temperature gradient $G_{S}$ in the crystal versus solute concentration $C_{L}$ in the melt for a pulling speed $V=1 \mathrm{~mm} / \mathrm{min}$ for $\mathrm{B}, \mathrm{P}$, and As. The dashed lines were calculated after Eq. (5) for the purely diffusive case. The solid lines were calculated after Eq. (9) for the case with melt convection assuming a rotation rate of the crystal of $\omega=10 \mathrm{rpm}$. The symbols highlight the critical temperature gradients for the case with melt convection and a solute concentration $C_{L}=1 \times 10^{20}$ atoms $/ \mathrm{cm}^{-3}$ at the beginning of the body growth and the corresponding values at a solidified fraction $g$ of $80 \%$. The error bars are due to the uncertainty of the material properties, especially for the diffusion coefficient $D$ used for the calculation of the temperature gradient $G_{S}$.

TABLE I

Material properties of silicon used to calculate the critical temperature gradient $G_{S}$ after Eq. (5) and Eq. (9).

\begin{tabular}{l|l}
\hline \hline Kinematic viscosity $\nu$ & $3 \times 10^{-7} \mathrm{~m}^{2} / \mathrm{s}$ \\
Thermal expansion $\beta$ & $1.4 \times 10^{-4} \mathrm{~K}^{-1}$ \\
Melting point $T_{\mathrm{m}}$ & $1683 \mathrm{~K}$ \\
Thermal conductivity (solid) $\lambda_{S}$ & $18.9 \mathrm{~W} /(\mathrm{m} \mathrm{K})$ \\
Thermal conductivity (liquid) $\lambda_{L}$ & $60.0 \mathrm{~W} /(\mathrm{m} \mathrm{K})$ \\
Latent heat $L$ & $1.8 \times 10^{6} \mathrm{~J} / \mathrm{kg}$ \\
Density $\rho$ & $2520 \mathrm{~kg} / \mathrm{m}^{3}$
\end{tabular}

The material properties of silicon and the physical properties of the dopants are compiled in Table I and Table II. For the calculations the D values given in the third raw were used which are within the published range. It is obvious that the smaller the segregation coefficient $k$ the higher must the temperature gradient $G_{S}$ in the crystal be to avoid constitutional supercooling. For a concentration $C_{L}=1 \times 10^{20}$ atoms $/ \mathrm{cm}^{-3}$ in the melt and a pulling speed $V=1 \mathrm{~mm} / \mathrm{min}$ the temperature gradient $G_{S}$ in the crystal must exceed $45 \mathrm{~K} / \mathrm{cm}$ for boron $\left(k_{\mathrm{B}}=\right.$ $\left.0.8 ; D_{\mathrm{B}}=2.4 \times 10^{-4} \mathrm{~cm}^{-2} / \mathrm{s}\right), 69 \mathrm{~K} / \mathrm{cm}$ for phosphorus

TABLE II

Segregation coefficient $k$ and diffusion coefficient $D$ of $B, P$, and $A$ s in silicon after $[6,22]$. In the third raw the $D$ values used in the present calculations are given.

\begin{tabular}{c|c|c|c}
\hline \hline Dopant & Boron & Phosphorus & Arsenic \\
\hline$k$ & 0.8 & 0.35 & 0.3 \\
$D\left[\mathrm{~m}^{2} / \mathrm{s}\right]$ & $1.2-2.4 \times 10^{-8}$ & $2.0-5.1 \times 10^{-8}$ & $1.9-3.3 \times 10^{-8}$ \\
$D_{\text {cal }}\left[\mathrm{m}^{2} / \mathrm{s}\right]$ & $2.4 \times 10^{-8}$ & $3.0 \times 10^{-8}$ & $2.7 \times 10^{-8}$
\end{tabular}


$\left(k_{\mathrm{P}}=0.35 ; D_{\mathrm{P}}=3.0 \times 10^{-4} \mathrm{~cm}^{-2} / \mathrm{s}\right)$ and $79 \mathrm{~K} / \mathrm{cm}$ for arsenic $\left(k_{\mathrm{As}}=0.3 ; D_{\mathrm{As}}=2.7 \times 10^{-4} \mathrm{~cm}^{-2} / \mathrm{s}\right)$. Thereby, it must be taken into account that the values of the diffusion coefficients $D$ reported for the various doping elements in literature can vary strongly depending of the doping element [6, 22], for example in case of phosphorus between $2.0-5.1 \times 10^{-4} \mathrm{~cm}^{-2} / \mathrm{sec}$ (see Table II). This results in an error of the calculation of the critical temperature gradient $G_{S}$. For simplicity, an average error of $+/-10 \%$ independent on the doping element was assumed to plot the error bars in Fig. 5 .

2.2.2. With convective species transport in the meltHurle's formula

In Czochralski crystal growth the transport conditions in the melt are typically dominated by convective melt flow [23]. In order to take into account convective solute transport in the melt Hurle modified Eq. (3) by implementing an effective segregation coefficient $k_{\text {eff }}$, see Eq. (6):

$$
\frac{G_{L}}{V}=\frac{(1-k)(-m) C_{L}}{k D} k_{\text {eff }}
$$

In a simple approximation the effective segregation coefficient $k_{\text {eff }}$ can be estimated according to Burton et al. [24]:

$$
k_{\text {eff }}=\frac{k}{k+(1-k) \exp (-V \delta / D)} .
$$

$\delta$ is a free parameter which can be estimated by a boundary layer thickness (compare Fig. 4a). The melt-crystal interface of a rotating Czochralski crystal can be approximated by a rotating disk in contact with a liquid. The boundary layer thickness $\delta$ of such a rotating disk is given according to Cochran [25] by

$$
\delta=1.6 D^{1 / 3} \nu^{1 / 6} \omega^{-1 / 2}
$$

with kinematic viscosity of the melt $\nu$ and rotation rate of the disk (= crystal) $\omega$. Using Eqs. (1), (3), and (6)-(8) one can derive an expression similar to Eq. (5) for the ratio of the temperature gradient $G_{S}$ in the crystal versus the pulling speed $V$ in dependence on the doping level in the melt $C_{L}$ for the case including convective melt flow

$$
\frac{G_{S}}{V}=\frac{\lambda_{L}}{\lambda_{S}} \frac{(1-k)(-m)}{k D} C_{L} k_{\text {eff }}+\frac{L \rho}{\lambda_{S}} .
$$

Assuming a typical value of the crystal rotation $\omega=$ $10 \mathrm{rpm}$ and a pulling speed $V=1 \mathrm{~mm} / \mathrm{min}$ the critical temperature gradient in the crystal $G_{S}$ calculated according to Eq. (9) in dependence of the doping concentration in the melt $C_{L}$ is shown in Fig. 5 by the solid lines for $\mathrm{B}, \mathrm{P}$, and As. It is obvious that under consideration of a melt flow $G_{S}$ is reduced compared to the case without melt flow and must exceed for an initial doping concentration $C_{L}=1 \times 10^{20} \mathrm{~cm}^{-3}$ only $44 \mathrm{~K} / \mathrm{cm}$ for boron (98\% of pure diffusive case), $51 \mathrm{~K} / \mathrm{cm}$ for phosphorus $(75 \%)$ and $54 \mathrm{~K} / \mathrm{cm}$ for arsenic $(68 \%)$ as shown by the symbols in Fig. 5. This phenomenon is well known that the smaller the segregation coefficient $k$, the more sensitive the solute transport depends on convection.

\section{Phenomena and parameters affecting constitutional supercooling during $\mathrm{CZ}$ growth of heavily doped silicon crystals}

\subsection{Enrichment of dopants in the melt due to segregation}

During pulling of the crystal the solute (dopant) will be rejected into the melt as all relevant dopants have $k<1$. Therefore, an increase of the solute concentration in the melt will take place with increasing process time. Assuming that the system is conservative, i.e. no solute is lost or added during growth and assuming total mixing by convection, the solute concentration in the crystal respectively in the melt can typically be described by Scheil's equation [26] in dependence of the solidified fraction $g$ :

$$
C_{L}=C_{0}(1-g)^{k-1} \text {. }
$$

We assume that at the beginning of the growth of the cylindrical crystal body the solute concentration in the melt is $C_{0}=1 \times 10^{20} \mathrm{~cm}^{-3}$. The growth of the whole cylindrical crystal body shall correspond to a solidified fraction $g$ of $80 \%$. This means that the concentration of the solute $C_{L}$ in the melt would increase from $C_{0}=1 \times 10^{20} \mathrm{~cm}^{-3}$ to $1.4 \times 10^{20} \mathrm{~cm}^{-3}$ for boron, to $2.9 \times 10^{20} \mathrm{~cm}^{-3}$ for phosphorus and to $3.1 \times 10^{20} \mathrm{~cm}^{-3}$ for arsenic at a solidified fraction $g=0.8$. As it is shown by the symbols in Fig. 5, this would theoretically require an increase of the critical temperature gradient $G_{S}$ in the crystal from $44 \mathrm{~K} / \mathrm{cm}$ at the start of the cylindrical body to $45 \mathrm{~K} / \mathrm{cm}$ at the end of the body for boron, from $51 \mathrm{~K} / \mathrm{cm}$ to $72 \mathrm{~K} / \mathrm{cm}$ for phosphorus and from $54 \mathrm{~K} / \mathrm{cm}$ to $83 \mathrm{~K} / \mathrm{cm}$ for arsenic. Alternatively, the pulling velocity $V$ has to be lowered from $V=1 \mathrm{~mm} / \mathrm{min}$ down to $0.97 \mathrm{~mm} / \mathrm{min}, 0.72 \mathrm{~mm} / \mathrm{min}$, and $0.67 \mathrm{~mm} / \mathrm{min}$ for $\mathrm{B}$, $\mathrm{P}$, and As respectively, while keeping the critical temperature gradient $G_{S}$ in the crystal constant from the beginning $(g=0)$ to the end $(g=0.8)$ of the cylindrical body.

Thereby, it must be stated that for boron these theoretical changes of either the temperature gradient or the pulling speed due to the segregation effect are negligible within the limits of error. Furthermore, the theoretically determined changes for $\mathrm{P}$ and As do not correspond to the real situation of $\mathrm{CZ}$ growth, because both solutes ( $\mathrm{P}$ and $\mathrm{As}$ ) are evaporating quantitatively from the silicon melt during growth. The evaporation rate depends on the argon pressure inside the puller [27]. Therefore, the evaporation of the volatile $\mathrm{P}$ and As can compensate the above segregation effect. This phenomenon can be used to control the homogeneity of the doping distribution by properly adjusting the argon pressure, respectively, the argon flow along the free melt surface during CZ pulling [28]. This effect of evaporation can be taken into account in the Scheil equation (Eq. (10)) by an additional parameter $k_{\mathrm{ev}}[27]$ :

$$
C_{L}=C_{0}(1-g)^{k+k_{\mathrm{ev}}-1} \text {. }
$$


e.g. $[11,27,29-31]$ and thus to calculate the criterion for constitutional supercooling taking into account segregation and evaporation.

\subsection{Crystal orientation}

Usually the [100] and [111] orientation are used in CZ growth of heavily doped silicon crystals. In case of the [111]-orientation a central facet can form which exhibits a higher doping concentration than the non-facetted periphery which grows with an atomically rough interface [32]. The higher doping of the central region for the growth with the facetted $\{111\}$ interface is explained by the higher effective segregation coefficient $k_{\text {fac }}[33]$. For $\mathrm{P}, k_{\mathrm{fac}}$ is up to $0.43(k=0.3)$ and for As is 0.41 $(k=0.35)$ [33]. Unfortunately no value of $k_{\text {fac }}$ for B was found in literature. By replacing $k$ by $k_{\mathrm{fac}}$ in Eq. (9) the critical temperature gradient $G_{S}$ can be estimated for a facetted interface. In case of phosphorus the critical temperature gradient $G_{S}$ becomes theoretically $49 \mathrm{~K} / \mathrm{cm}$ for the facetted interface instead of $51 \mathrm{~K} / \mathrm{cm}$ for the rough interface and in case of arsenic $G_{S}$ is $51 \mathrm{~K} / \mathrm{cm}$ for the facetted interface instead of $54 \mathrm{~K} / \mathrm{cm}$ for the non-facetted one. These changes are within the error limits of the model assumptions. Therefore, it can be concluded that the presence of a facetted interface in case of pulling a [111]-oriented crystal has a negligible influence on the stability limit for constitutional supercooling.

\subsection{Pulling rate and variations thereof}

During a typical Czochralski growth run of silicon the pull rate $V$ differs significantly during the different process steps. This is illustrated by Fig. 6 where the pulling rate $V$ and the crystal diameter are displayed during a typical CZ growth run of a silicon crystal with a diameter of $100 \mathrm{~mm}$ of the cylindrical body [34]. At the beginning the pull rate $V$ is increased up to several $\mathrm{mm} / \mathrm{min}$ during the necking procedure called after Dash [35]. Then, $V$ is reduced to enlarge the crystal diameter during growth of the starting cone. During shoulder growth, i.e. the transition from the starting cone to the cylindrical crystal body, $V$ is increased again to counteract an uncontrolled increase of the crystal diameter. In the present case the pull rate jumps from $0.5 \mathrm{~mm} / \mathrm{min}$ during growth of the middle part of the shoulder to around $1.7 \mathrm{~mm} / \mathrm{min}$ at the end of the shoulder. During growth of the cylindrical crystal body $V$ is on average around $1.2 \mathrm{~mm} / \mathrm{min}$ and its variations are relatively small, nevertheless with $V$ as parameter for controlling and achieving a $\mathrm{CZ}$ crystal with a constant diameter. The crystal diameter is decreased at the end ("end cone") by a slight increase of the pull rate compared to the value during body growth, before the crystal is finally detached from the melt.

As the critical temperature gradient $G_{S}$ in the crystal is nearly proportional to the pull rate $V$ (according to Eq. (9)), $G_{S}$ must be expected to vary also significantly during the different process phases of a typical $\mathrm{CZ}$ growth run as shown in Fig. 6. This is illustrated by Fig. 7 where

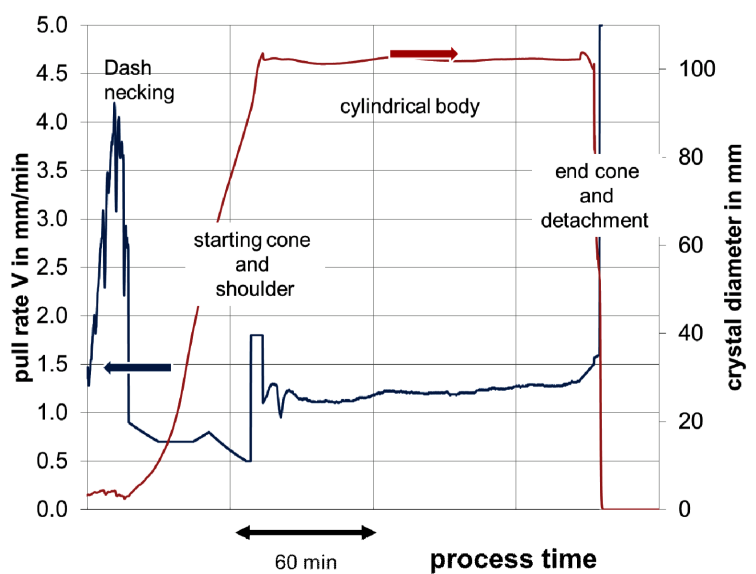

Fig. 6. Pull rate $V$ (left ordinate) and crystal diameter (right ordinate) as detected by the control system versus process time for $\mathrm{CZ}$ pulling of a silicon crystal with a diameter of $100 \mathrm{~mm}$ in the cylindrical part (body) [35].

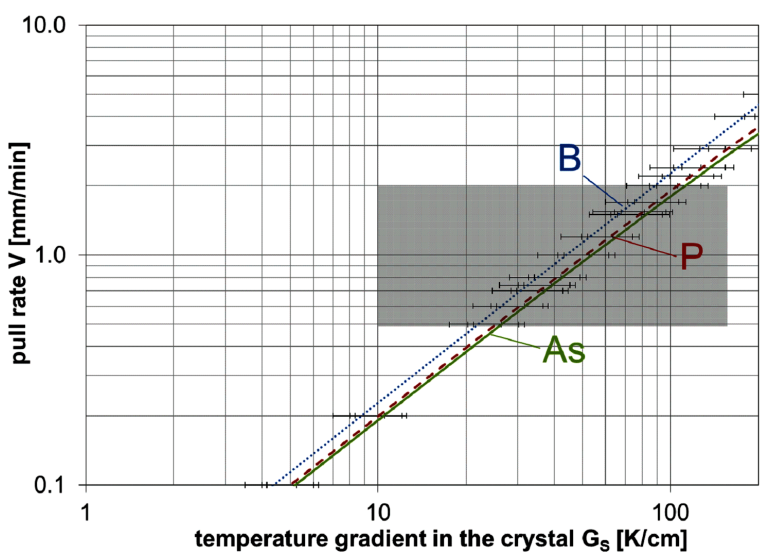

Fig. 7. Pull rate $V$ versus critical temperature gradient $G_{S}$ in the crystal computed after Eq. (9) for a rotation rate of the crystal $\omega=10 \mathrm{rpm}$ and a solute concentration in the melt $C_{L}=1 \times 10^{20}$ at. $/ \mathrm{cm}^{-3}$ for B (dotted line), $\mathrm{P}$ (dashed line), and As (solid line). The grey area marks typical pulling rates which are used to grow silicon crystals with diameters of 100-200 mm (compare Fig. 6). The error bars are due to the uncertainty of the material properties, especially for the diffusion coefficient $D$ used for the calculation of the temperature gradient $G_{S}$

the relationship between the pulling rate $V$ and the critical temperature gradient $G_{S}$ in the crystal is plotted for B, P, and As. The curves were computed by Eq. (9) for an initial doping concentration of $1 \times 10^{20} \mathrm{~cm}^{-3}$ in the melt. If one assumes a pull speed $V$ of the cylindrical body of around $1 \mathrm{~mm} / \mathrm{min}$, then the required critical temperature gradient $G_{S}$ in the crystal is at least $100 \%$ higher during the Dash necking $\left(V_{\text {neck }}>2 \mathrm{~mm} / \mathrm{min}\right)$ than during growth of the crystal body. The growth of the starting cone and the transition from the starting cone to the cylindrical body seem to be also very critical with re- 
spect to constitutional supercooling. The high values of the pull rate $V$ which are used during this process phase are corresponding to large values of the critical temperature gradient $G_{S}$, which should be at least $70 \%$ higher than for the growth of the body in the given case. Finally, it can be assumed that the pulling of the end cone is critical, too. The solute has enriched in the remaining shallow (poorly mixed) melt and the pulling speed is increased to decrease the crystal diameter. However, no quantitative data neither for the pulling speed nor for the actual temperature gradient in the crystal are available for the region of the end cone. Therefore, the growth of the end cone cannot be considered in the further discussion.

\section{Discussion}

In this chapter an attempt is undertaken to discuss the limitations of $\mathrm{CZ}$ growth of heavily doped silicon crystals with respect to constitutional supercooling under "industry-like" conditions. The problem of such a consideration is the fact that temperature distribution in a $\mathrm{CZ}$ puller and therefore in the crystal is depending strongly on the actual hot-zone design and process parameters which are usually confidential in industrial production. However, there are some reports $[36-42]$ and also references therein where the temperature distribution in the crystal for different puller geometries and process conditions have been analyzed. Furthermore, studies exist about modeling of the heat transfer in $\mathrm{CZ}$ pulling of silicon crystals. These published experimental and numerical results are used in the following in order to estimate the range of the typical temperature gradient in the crystal $G$ during the different process phases and for different crystal diameters. These ranges of temperature gradients will be compared to the critical temperature gradients $G_{S}$ which are needed to avoid constitutional supercooling.

Table III shows the results of such a quantitative comparison. Column 1 denotes the different $\mathrm{CZ}$ process phases according to Fig. 6. Column 2 contains the realistic pulling rates $V$ in the three different process phases which were used to calculate by Eq. (9) the corresponding critical temperature gradients $G_{S}$ which are needed to avoid constitutional supercooling for a B, P and As concentration in the melt $C_{L}=1 \times 10^{20} \mathrm{~cm}^{-3}$ (columns 4,5 and 6 in Table III). The actual temperature gradients $G$ which are given in column 3 were estimated from published experimental and simulation data as follows.

The temperature gradient $G$ in the crystal during the seeding and necking phase is in the order of $50-100 \mathrm{~K} / \mathrm{cm}$ according to direct measurements performed in a $\mathrm{CZ}$ puller for growing silicon crystals with $300 \mathrm{~mm}$ diameter [42] and according to experiments [36, 40] with smaller crystal diameters. For the growth of the cylindrical body $G$ is typically decreased with increasing crystal diameter regardless of the doping level and the actual hot-zone design. The reported values of $G$ are in the range of 20$60 \mathrm{~K} / \mathrm{cm}$ for crystals with $100 \mathrm{~mm}$ and $200 \mathrm{~mm}$ diameter
TABLE III

Available data in literature about pulling rates $V$ (column 2 ) and temperature gradients $G$ (column 3 ) used for certain CZ process phases: necking, starting cone and shoulder, and body growth of silicon crystals with diameters of 100 $200 \mathrm{~mm}$. The last three columns denoted by B, P, and As contain the critical temperature gradients $G_{S}$ calculated after Eq. (9) using the values of $V$ from column 2 for a solute concentration $C_{L}=1 \times 10^{20}$ at. $/ \mathrm{cm}^{3}$.

\begin{tabular}{c|c|c|c|c|c}
\hline \hline 1 & 2 & 3 & $\mathrm{~B}$ & $\mathrm{P}$ & $\mathrm{As}$ \\
\hline Process phase & $V[\mathrm{~mm} / \mathrm{min}]$ & $G[\mathrm{~K} / \mathrm{cm}]$ & \multicolumn{3}{|c}{$G_{S}[\mathrm{~K} / \mathrm{cm}]$} \\
\hline necking & $>2$ & $50-100$ & 88 & 106 & 112 \\
shoulder & $0.5-1.5$ & $25-80$ & $22-60$ & $25-78$ & $26-82$ \\
body & $0.8-1.2$ & $20-60$ & $35-53$ & $40-62$ & $43-65$
\end{tabular}

at pulling rates $V$ of around $1 \mathrm{~mm} / \mathrm{min}[39,40,43]$. The temperature gradient can also vary along the length of the cylindrical body as reported e.g. in [40]. For the transition from the starting cone to the cylindrical body it is concluded from very few experimental data $[39,40]$ that $G$ is about $10-50 \%$ higher than in the subsequent cylindrical body phase. More precise data could only be obtained by fully time-dependent simulations which are still very challenging [23].

In the criterion of constitutional supercooling (according to Fig. 4b and Eq. (3)) the actual temperature gradient $G$ has to be taken in a crystal position very close to the growth interface. Measured temperature gradients represent usually values which are either averaged over the whole crystal length or which are determined at a certain distance away from the solid-liquid interface. However, it is known from numerical simulations that the temperature gradient can be e.g. $20 \%$ to $30 \%$ higher directly at the interface than at a distance of a few centimeters away from the phase boundary [38, 43]. This means that the actual $G$ values are most likely higher than the $G$ values given in Table III.

In any case it follows clearly from a comparison of columns 3 and 4-6 in Table III that the avoidance of constitutional supercooling during growth of heavily doped silicon crystals is very challenging. The critical temperature gradients $G_{S}$ which are needed for a stable growth are very close to the upper limit of the values of the temperature gradients $G$ which are reported in the literature for various $\mathrm{CZ}$ growth conditions. If one considers the different doping elements it is also obvious that the avoidance of constitutional supercooling is more difficult for growth of As-doped Si crystals than for P-and B-doped ones (compare columns 4, 5 and 6 in Table III).

An increase of the crystal diameter (e.g. from $100 \mathrm{~mm}$ to $200 \mathrm{~mm}$ ) would intensify the problem because it has to be considered that for a given hot-zone typically the temperature gradient $G$ decreases with increasing crystal diameter [44]. Furthermore, it has to be considered that the axial temperature gradient $G$ varies along the radial position. It is typically higher at the periphery of the crystal compared to the center [43]. This means 
that a breakdown of the interface caused by constitutional supercooling will preferentially begin in the core of the crystal rather than at its periphery. This prediction is confirmed by several experimental results $[3,7-15]$.

From the arguments above, it can be easily understood that the growth of silicon crystals heavily doped with $\mathrm{P}$ or As with $300 \mathrm{~mm}$ diameter would be extremely difficult. Although the pulling speed $V$ is typically only $0.5-0.6 \mathrm{~mm} / \mathrm{min}$ for shallow doped crystals with $300 \mathrm{~mm}$ diameter during the growth of the cylindrical body $[45,46]$, the temperature gradients $G$ reported in literature are also only in the order of $30-40 \mathrm{~K} / \mathrm{cm}$ during body growth [45]. This means that the growth of silicon crystals with $300 \mathrm{~mm}$ diameter would take place again very close to the criterion of constitutional supercooling for $\mathrm{P}$ and As (see Fig. 7). In addition, it has to be considered that the larger mass of the melt $(>250 \mathrm{~kg}$ typically used for $300 \mathrm{~mm}$ diameter instead of $30 \mathrm{~kg}$ for $100 \mathrm{~mm} \mathrm{[46])} \mathrm{would} \mathrm{require} \mathrm{the} \mathrm{handling} \mathrm{of} \mathrm{ten} \mathrm{times}$ more mass of the hazardous phosphorus and arsenic. This is extremely challenging from a safety and security point of view. For silicon heavily doped with $B$ the growth of crystals with $300 \mathrm{~mm}$ diameter might become more feasible from both, the occurrence of constitutional supercooling and the doping issues - independently of economic considerations.

\section{Conclusions and recommendations}

It has been shown in the previous sections that it is very challenging to grow silicon crystals by the $\mathrm{CZ}$ technique with high doping levels $\left(>10^{20}\right.$ atoms $\left./ \mathrm{cm}^{3}\right)$ of $\mathrm{B}$, $\mathrm{P}$, and As without running into the problem of constitutional supercooling followed by the "loss of structure".

From the theoretical background one can conclude that the following measures should be considered to avoid constitutional supercooling:

- optimization of the hot-zone of the puller e.g. by introducing additional heat shields in order to establish a higher temperature gradient $G$;

- decrease of the pulling speed $V$ in order to decrease the pile up of doping atoms in the melt in front of the growth interface;

- increased convective melt transport in order to decrease the pile up of doping atoms in the melt in front of the growth interface.

On the other hand, for economic reasons it is not desirable to decrease the pulling rate $V$. Moreover, an increase of the temperature gradient $G$ would result in an increase of the thermal stress in the crystal [44]. This could lead to the formation of crystal defects, like dislocations and finally structure loss even without violating the criterion for constitutional supercooling.

It is also discussed in literature that the structure loss during $\mathrm{CZ}$ growth of heavily doped silicon crystals may depend on the concentrations of oxygen and intrinsic point defects, e.g. $[6,12,15]$. So far there are only a few publications et al. [47-50] in which the influence of doping on the concentration of intrinsic point defects is studied. It is discussed that high doping concentrations of $\mathrm{B}, \mathrm{P}$, and As can have a considerable influence on the formation of three-dimensional crystal defects like voids, crystal originated particles (COPs), oxygen precipitates and the position of the oxygen induced stacking fault (OFS) ring [47-50]. However, no clear picture exists so far in literature especially regarding the correlation of the occurrence of structure loss with the point defect content of heavily doped silicon crystals.

Therefore, it will remain difficult to prove in a certain case whether constitutional supercooling is responsible for a "structure loss" or whether other phenomena were limiting the dislocation-free growth of heavily doped silicon crystals by the Czochralski technique.

\section{Acknowledgments}

The authors would like to thank Dr. Lothar Lehmann from Siltronic AG for fruitful discussions. The research and development activities were carried out within the Power-on-Si Project (project number: 100128500) with Siltronic AG. The project is funded partly by the European Regional Development Fund (ERDF) and by the Saxony State Ministry for Science and Art (SMWK).

\section{References}

[1] B. Jayant Baliga, Advanced Power MOSFET Concepts, Springer, New York 2010, Ch. XVI.

[2] www.semi.org; SEMI Document 5307, 2011.

[3] H.D. Chiou, J. Electrochem. Soc. 147, 345 (2000).

[4] M. Kulkarni, M. Banan, C. Luers, US-Patent 7131 091 B2, 2006.

[5] R. Falster, V. Voronkov, G. Borionetti, US-Patent 8 026145 B2, 2011.

[6] R. Scala, M. Porrini, G. Borionetti, Cryst. Res. Technol. 46, 749 (2011).

[7] W. Bardsley, J.M. Callan, H.A. Chedzey, D.T.J. Hurle, Solid State Electron. 3, 142 (1961).

[8] K.M. Kim, J. Electrochem. Soc., Solid State Sci. Technol. 126, 875 (1979).

[9] W. Bardsley, D.T.J. Hurle, M. Hart, A.R. Lang, J. Cryst. Growth 49, 612 (1980).

[10] T.G. Digges, R. Shima, J. Cryst. Growth 50, 865 (1980).

[11] H.M. Hobgood, T.T. Braggins, M.M. Sopira, J.C. Swartz, R.N. Thomas, IEEE Trans. Electron Dev. 27, 14 (1980).

[12] H.D. Chiou, Electrochem. Soc. Proc. 99, 511 (1999).

[13] T. Taishi, X. Huang, M. Kubota, T. Kajigaya, T. Fukami, K. Hoshikawa, Jpn. J. Appl. Phys. 38, 223 (1999).

[14] T. Taishi, X. Huang, M. Kubota, T. Kajigaya, T. Fukami, K. Hoshikawa, Mater. Sci. Eng. B 72, 169 (2000). 
[15] H.D. Chiou, J. Electrochem. Soc. 152, G295 (2005).

[16] J.W. Rutter, B. Chalmers, Can. J. Phys. 31, 15 (1953).

[17] A. Cröll, University Freiburg, private communication.

[18] W.W. Mullins, R.F. Sekerka, J. Appl. Phys. 35, 444 (1964).

[19] W.A. Tiller, K.A. Jackson, J.W. Rutter, B. Chalmers, Acta Metall. 1, 428 (1953).

[20] D.T.J. Hurle, Solid State Electron. 3, 37 (1961).

[21] C.D. Thurmond, M. Kowalchik, Bell Syst. Tech. J. 39, 169 (1960).

[22] A.G. Ostrogorsky, J. Cryst. Growth, accepted for publication.

[23] G. Müller, J. Friedrich, J. Cryst. Growth 266, 1 (2004).

[24] J.A. Burton, R.C. Prim, W.P. Slichter, J. Chem Phys. 21, 1987 (1953).

[25] W.G. Cochran, Math. Proc. Cambr. Philos. Soc. 30, 365 (1934).

[26] E. Scheil, Z. Metallkunde 34, 70 (1942).

[27] S.E. Bkadshaw, A.I. Mlavsky, J. Electron. Control 2, 134 (1956).

[28] W. Zulehner, J. Cryst. Growth 65, 189 (1983).

[29] T.F. Ciszek, J. Cryst. Growth 75, 61 (1986).

[30] Z. Liu, T. Carlberg, J. Electrochem. Soc. 140, 2052 (1993).

[31] A.Ya. Nashelsky, E.O. Pulner, J. Cryst. Growth 171 , 94 (1997).

[32] J.C. Brice, J. Cryst. Growth 6, 205 (1970).

[33] K.T. Wilke, J. Bohm, Crystal Growth (Kristallzüchtung), Harry Deutsch Verlag, Frankfurt 1988 (in German).

[34] A. Molchanov, PVA Tepla AG, private communication.

[35] W. Dash, J. Appl. Phys. 30, 459 (1959).

[36] E. Kuroda, H. Kozuka, J. Cryst. Growth 63, 276 (1983).
[37] T. Fujiwara, S. Inami, S. Miyahara, S. Kobayashi, T. Kubo, H. Fujiwara, J. Cryst. Growth 128, 275 (1993).

[38] E. Dornberger, E. Tomzig, A. Seidl, S. Schmitt, H.-J. Leister, Ch. Schmitt, G. Müller, J. Cryst. Growth 180, 461 (1997).

[39] H.D. Chiou, T.-Y.T. Lee, S. Teng, J. Electrochem Soc. 144, 2881 (1997).

[40] T. Abe, in: Defects in Silicon III, Eds. T. Abe, W.M. Bullis, S. Kobayashi, W. Lin, P. Wagner, Electrochemical Society Proceeding, Vol. 99-1, Seattle 1999, p. 414.

[41] X. Huang, T. Taishi, T. Wang, K. Hoshikawa, J. Cryst. Growth 229, 6 (2001).

[42] N. Banos, M.Sc. Thesis, University Erlangen Nuremberg 2002 (in German)

[43] E. Dornberger, W. von Ammon, J. Virbulis, B. Hanna, T. Sinno, J. Cryst. Growth 230, 291 (2001).

[44] W. von Ammon, E. Dornberger, P.O. Hansson, J. Cryst. Growth 198/199, 390 (1999).

[45] M. Krause, J. Friedrich, G. Müller, Mater. Sci. Semicond. Proc. 5, 361 (2003).

[46] W. von Ammon, in: Crystal Growth - from Fundamental to Technology, Eds. G. Müller, J.J. Metois, P. Rudolph, Elsevier, Amsterdam 2004, p. 239.

[47] V.V. Voronkov, B. Falster, Microelectron. Eng. 56 , 165 (2001).

[48] V.V. Voronkov, R. Falster, M. Porrini, J. Duchini, Phys. Status Solidi A 209, 1898 (2012).

[49] M. Suhren, D. Graef, U. Lambert, P. Wagner, J. Electrochem. Soc. 144, 4041 (1997).

[50] W. Sugimura, T. Ono, S. Umeno, M. Hourai, K. Sueoka, K. Koji, Electrochem. Soc. Trans. 2, 95 (2006). 